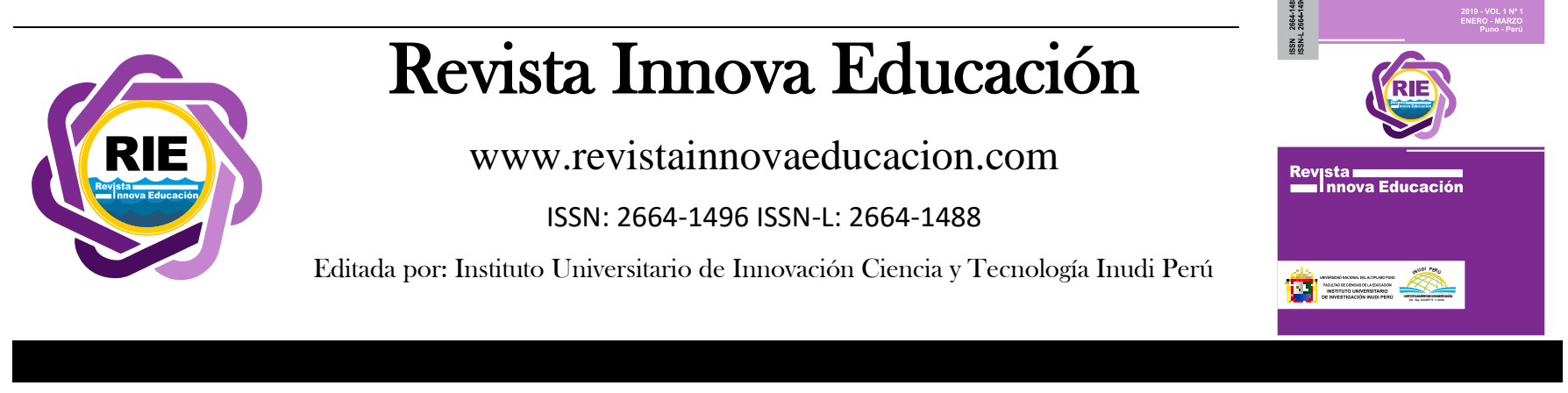

\title{
Análisis multidimensional de la pertinencia. Estudio de caso de un modelo de intervención con enfoque diferencial
}

\author{
Multidimensional analysis of relevance. Case study of an intervention model with a differential \\ approach
}

\author{
Gustavo Rincón ${ }^{1 *}$ \\ https://orcid.org/0000-0001-9683-3637
}

\author{
Nathaly Jimenez** \\ https://orcid.org/0000-0001-9521-1246
}

\author{
Michelle Castrillon** \\ https://orcid.org/0000-0003-2467-8361
}

DOI: https://doi.org/10.35622/j.rie.2020.01.006

* Servicio Nacional de Aprendizaje SENA, Bogotá - Bogotá, Colombia

** Universidad del Valle, Bogotá - Bogotá, Colombia

Recibido el 18/12/2019/ Aceptado el 27/01/2020 Publicado el 31/01/2020

\begin{tabular}{ll}
\hline ARTíCULO ORIGINAL & $\begin{array}{l}\text { La literatura ha demostrado la relevancia que tiene la educación no solo sobre el } \\
\text { desarrollo económico de una sociedad, sino también en el desarrollo humano y cultural } \\
\text { de los individuos. Es por esto, que uno de los principales retos de los gobiernos es } \\
\text { garantizar el acceso de los individuos de diferentes grupos poblacionales a la educación }\end{array}$ \\
$\begin{array}{l}\text { Equidad social, } \\
\text { formación para el } \\
\text { trabajo, pertinencia, } \\
\text { población expuesta al } \\
\text { conflicto, población } \\
\text { vulnerable. }\end{array}$ & $\begin{array}{l}\text { y su calidad, buscando que esta sea pertinente según el contexto económico, histórico, } \\
\text { social y cultural. En este sentido, este documento busca analizar la pertinencia de } \\
\text { algunos programas de formación ofertados bajo un modelo de intervención focalizado }\end{array}$ \\
& $\begin{array}{l}\text { a atender población vulnerable o expuesta al conflicto, sobre la base de un conjunto de } \\
\text { factores relevantes para el desarrollo del individuo y de la sociedad, implementado un } \\
\text { enfoque multidimensional que comprende lo educativo-pedagógico, lo social en } \\
\text { conjunto con la equidad Social y cultural y, por último, lo económico, institucional y } \\
\text { político. Este análisis se complementa con la descripción del comportamiento de los } \\
\text { aprendices, específicamente en sus actitudes cooperativas y los sesgos integrales, a } \\
\text { partir de la aplicación de técnicas de economía experimental y del comportamiento. }\end{array}$ \\
\hline
\end{tabular}

\section{KEYWORDS}

Social equity, job training, relevance, population exposed to
The academic literature has demonstrated the relevance of education not only on the economic development of society but also on the human and cultural development of individuals. That is why, one of the main challenges of governments is the access of individuals of different population groups to education and its quality, the search for the relevant sea according to the economic, historical, social and cultural context. In this sense, this document seeks to analyze the relevance of some training programs

\footnotetext{
${ }^{1}$ Correspondencia: grincon54@misena.edu.co
} 
conflict, vulnerable population. offered under an intervention model focused on the population, based on the set of factors important for the development of the individual and society, implemented a multidimensional approach that includes the educational-pedagogical, the social in conjunction with social and cultural equity, finally, the economic, institutional and political. This analysis is complemented by the description of the behavior of the apprentices, specific in their cooperative attitudes and integral biases, based on the application of experimental and behavioral economics techniques.

\section{INTRODUCCIÓN}

La firma del acuerdo de paz en el 2016 trajo consigo una serie de retos para el Estado Colombiano, como promover la integración de las regiones, el desarrollo social, económico y equitativo del país, así como la inclusión integral de los excombatientes. En este sentido, el fortalecimiento de la educación en términos de acceso y calidad es parte fundamental en dicho proceso de inclusión, pues la educación representa uno de los mecanismos esenciales para contribuir, no solo al desarrollo económico sino, al crecimiento y fortalecimiento personal y en ultimas al desarrollo de la sociedad, al trasmitir también sus valores y cultura. (Malagón, 2006; UNESCO, 2008).

En este contexto, el Servicio Nacional de Aprendizaje SENA, como institución de formación para el trabajo y el desarrollo humano (Ley 1064, 2006) y en cumplimiento de su misión, se convierte en uno de los actores fundamentales para dinamizar las acciones propuestas en el acuerdo de paz. Por tal razón, el Centro de Gestión Tecnológica de Servicios (CGTS) ${ }^{2}$ Diseña, a partir del Modelo de Formación Profesional Integral (FPI ${ }^{3}$, el Modelo SENA de Intervención Integral (MSII) para promover el desarrollo humano y socioeconómico de población vulnerable y población expuesta al conflicto (excombatientes y víctimas del conflicto armado). Lo anterior, dada la necesidad de responder a la actual demanda social y a las posibles limitantes derivadas de condiciones asociadas a la pobreza y la desigualdad a los que se enfrenta esta población para desarrollar plenamente su proceso formativo, pues no cuentan con los recursos para acceder a herramientas de apoyo que les permita desarrollar sus competencias básicas (Terigi, 2009; Ramírez, 2010; Santos, 1998).

Ahora bien, El MSII consta de tres etapas, en las cuales los aprendices inician los programas de formación con un entrenamiento vocacional y técnico enfocado a los contenidos de

\footnotetext{
${ }^{2}$ El CGTS es uno de los centros de formación que hace parte del SENA. Se encuentra ubicado en Cali - Valle del Cauca e imparte formación técnica, tecnológica y complementaria, tanto presencial como virtual, en las áreas: Hotelería, Turismo y Gastronomía, Comercio, Administración y Finanzas, Salud y Servicios Personales, Actividad Física, Logística y Gestión de la Producción Industrial y Ambiental, atendiendo en su mayoría a jóvenes de poblaciones vulnerables y de bajos recursos de la ciudad y de municipios cercanos como Yumbo, Vijes, Tuluá, Dagua, Jamundí y La Cumbre.

${ }^{3}$ El Modelo de formación profesional integral implementado por el SENA es una propuesta pedagógica fundamentada el desarrollo humano de los aprendices proceso educativo teórico - práctico de carácter integral, mediante el cual la persona adquiere y desarrolla de manera permanente conocimientos, destrezas y aptitudes e identifica genera y asume valores y actitudes para su realización humana y su participación, crítica y creativa en el mundo social y productivo
} 
cada curso, lo cual está alineado con algunos proyectos educativos que se han desarrollado donde se observa que la educación tiene un papel fundamental en la reintegración a la vida civil de los excombatientes (Harris y Mische, 2004; Observatorio de Procesos de Desarme, Desmovilización y Reintegración, 2009;). Posteriormente, desarrollan un segundo componente que aborda temáticas de cultura de paz $^{4}$ que se complementa con un tercer componente que busca desarrollar habilidades blandas en los aprendices mediante el deporte, denominado DEPORPAZ. Este último componente consiste en la práctica específica del deporte "batalla de fuerza", que involucra dentro de sus características, elementos que favorecen el desarrollo de habilidades blandas convirtiéndose en una especie de mecanismo para reducir la brecha social entre los diferentes tipos de población y contribuir así a una efectiva reinserción de los excombatientes y víctimas (Diaz y González, 2001; Moscoso, Pérez, Muñoz, González, y Rodríguez, 2013; García, Gutiérrez, González y Valero, 2012).

Los programas que se ofertaron inicialmente bajo el MSII fueron programas de nivel técnico para los cuales se realizó una revisión del contexto, competencias a desarrollar y perfiles de egreso de cada programa, teniendo en cuenta que estos involucran una serie de competencias y resultados de aprendizaje que era necesario se ajustaran a la demanda social y a la historia de vida de la población objetivo, sin embargo posteriormente se ajustó la oferta y se agregaron cursos complementarios, dado que la población expresó tener inconvenientes respecto a la intensidad del horario de formación, cohibiéndolos de incorporarse al programa técnico.

Surge entonces, un reto adicional en el fortalecimiento de la educación relacionado directamente con su calidad, la pertinencia, pues es uno de los aspectos con los que se define el deber ser y la relevancia de la educación o en este caso particular, la formación para el trabajo. Sin embargo, la pertinencia ha sido un fenómeno difícil de abordar, según la UNESCO (1998) la pertinencia requiere la construcción de una visión conjunta de futuro y las necesidades del contexto, así mismo se debe ejecutar en función de las expectativas sociales y las acciones desarrolladas por la institución. De acuerdo con esto, las actividades desarrolladas deben estar encaminadas a la solución de problemas sociales, no solo enfocados a la modernización de la educación superior en el ámbito laboral o al suministro de recursos humanos calificados (Sutz, 1997; Gibbons, 1998), sino también en función de los inconvenientes subyacentes como la pobreza, la intolerancia, el respeto, el cuidado del medio ambientes, el fomento de la paz, entre otros.

\footnotetext{
${ }^{4} \mathrm{El}$ componente de cultura de paz se incluye en el modelo, dada la importancia de que las personas en formación estén contextualizadas sobre las realidades sociales, nacionales y globales y en esa medida aportar desde su área de conocimiento con acciones que generen impacto sobre su entorno inmediato (Higuera, 2018).
} 
Ahora bien, teniendo en cuenta que la educación promueve el desarrollo individual y social, la pertinencia de la educación también debe de involucrar en su análisis aspectos que aborden las necesidades en ambos sentidos (Martuscelli y Martínez, 2002; García, 2002; Malagón, 2009), así como encontrar un equilibrio entre las necesidades del sector productivo y la economía, las necesidades de la sociedad en su conjunto y del individuo como ser humano, dentro de un contexto histórico, económico, social y cultural (Tunnermman, 2000; Dias, 2008).

El presente documento busca analizar la pertinencia de los programas de formación ya mencionados, sobre la base de un conjunto de factores relevantes para el desarrollo del individuo y de la sociedad, implementado un enfoque multidimensional que comprende lo educativopedagógico, lo social en conjunto con la equidad Social y cultural y, por último, lo económico, institucional y político (Tunermann, 2000; Malagon, 2006). A su vez, este análisis se complementa con la descripción de los patrones de comportamiento de los aprendices que se derivaron de la intervención del MSII, específicamente en sus actitudes cooperativas y los sesgos integrales, teniendo presente la teoría de la identidad social planteada por Tajfel y Turner (1979) en la que determinan tres ideas principales que son la categorización, la identificación y la comparación, las cuales dan paso a que se generen estereotipos y favoritismos en una población.

En este sentido, este articulo consolida los resultados encontrados en el proyecto "Evaluación de dos componentes del modelo SENA de intervención integral para el desarrollo humano y socioeconómico de las personas en proceso de reincorporación y/o víctimas del conflicto armado ubicadas en el valle del cauca 2018" presentado a la convocatoria 2018 de Sennova, el cual tuvo como producto un libro $^{5}$ en el que se evalúa uno de dos componentes, la pertinencia. En este caso, los resultados detallados en dicho producto se complementan con lo encontrado en materia de comportamiento.

\section{MÉTODO Y MATERIALES}

Teniendo en cuenta que esta investigación busca analizar la pertinencia de los programas de formación para el trabajo bajo el MSII para la población vulnerable, excombatientes y víctimas del conflicto armado, la metodología implementada consiste en primera instancia, en realizar un análisis documental a diferentes fuentes secundarias en las que se define y analiza la pertinencia, con el fin de establecer las dimensiones sobre las cuales de desarrollará el análisis de la misma, siguiendo la metodología que sintetiza Peña (2006) para la implementación de esta técnica.

\footnotetext{
${ }^{5}$ El libro se denominó "Pertinencia de los programas académicos ofertados por el SENA a población reinsertada, reincorporada y víctimas del conflicto armado colombiano”, fue escrito por los instructores Gustavo Rincón y Jhon Jairo Angarita y publicado en el 2019.
} 
Por otro lado, para examinar la pertinencia en los programas objeto de estudio, se realizó un análisis cualitativo de datos colectados a partir de la implementación de entrevistas semiestructuradas a aprendices, instructores y directivos del CGTS teniendo como referente metodológico el ejercicio propuesto por Mendoza (2018) sobre la pertinencia social del programa de inclusión educativa para adultos en el municipio de Candelaria en el Valle del Cauca.

En segunda instancia se llevó a cabo un análisis cuantitativo de carácter descriptivo; la obtención estos datos se ejecutó a través de la aplicación de técnicas de economía experimental y del comportamiento a la población beneficiaria de los programas de formación, se corrieron sesiones en las que se desarrollaron juegos experimentales para medir sus actitudes cooperativas y sesgos.

El desarrollo de este análisis de datos se realizó para los programas ofertados en la implementación del MSII, en el cual se ejecutaron programas de nivel técnico (cuatro grupos) y cursos complementarios (10 grupos) facilitando la vinculación del tipo de población objetivo en 14 grupos con un promedio de 25 aprendices en cada uno. Los programas de nivel técnico fueron Operación de Alojamientos Rurales, Manejo Ambiental (dos grupos) y Ventas de Productos y Servicios (dos grupos) para un total de cuatro grupos, mientras que los cursos complementarios fueron Turismo (un grupo), Ventas (cuatro grupos) y Cocina (cinco grupos).

\section{Diseño metodológico}

Inicialmente, para poder establecer el análisis documental de información secundaria alrededor de la pertinencia social y educativa, se realizó una revisión del estado del arte, además de la construcción de un marco conceptual sobre el cual fue posible definir las categorías analíticas que permitieron describir la pertinencia de manera integral. A partir de dicha revisión, se toma como orientación principal, la concepción de Malagón (2006), que a su vez recoge lo expuesto por Tunnermann (2010) donde es claro que además de la relación de retroalimentación que debe existir entre las instituciones de educación y el sector productivo, estas dinámicas también deben de verse reflejadas en relación con el Estado, los sectores sociales y el sistema de valores de una sociedad. Por tal razón, se definieron las categorías descritas en la Tabla 1, para rastrear e ilustrar este concepto. 
Tabla 1. Categorías de análisis para la revisión documental

\begin{tabular}{|c|c|c|}
\hline $\begin{array}{c}\text { Enfoques } \\
\text { categoriales }\end{array}$ & Definición & Categorías \\
\hline Institucional & $\begin{array}{l}\text { Aborda el análisis de los procesos que desarrolla la } \\
\text { institución que imparte la formación, las ideas sobre su } \\
\text { función social, la disposición de recursos. etc. }\end{array}$ & $\begin{array}{l}\text { Proyecto Educativo } \\
\text { Institucional PEI } \\
\text { Objetivos } \\
\text { Diseño institucional }\end{array}$ \\
\hline Política & $\begin{array}{l}\text { Se centra en el compromiso o enfoque con la solución de los } \\
\text { problemas sociales. }\end{array}$ & $\begin{array}{l}\text { Investigación } \\
\text { Proyección Social }\end{array}$ \\
\hline $\begin{array}{l}\text { Educativo- } \\
\text { pedagógica }\end{array}$ & $\begin{array}{l}\text { Este está dirigido específicamente a la parte académica y } \\
\text { pedagógica, a los contenidos curriculares y la manera en que } \\
\text { se imparten. Cómo se aborda el proceso educativo y de } \\
\text { aprendizaje. }\end{array}$ & $\begin{array}{l}\text { Modelo pedagógico } \\
\text { Oferta académica } \\
\text { Metodologías }\end{array}$ \\
\hline Social & $\begin{array}{l}\text { Formación integral del estudiante, en relación con los } \\
\text { valores, la ética social, el sentido de pertenencia a una } \\
\text { comunidad, con lo humano }\end{array}$ & $\begin{array}{l}\text { Valores } \\
\text { Sentido de pertenencia } \\
\text { Habilidades blandas } \\
\text { Contexto del estudiante }\end{array}$ \\
\hline Equidad social & $\begin{array}{l}\text { Se basa en revisar las oportunidades que brinda para la } \\
\text { superación de las condiciones que obstaculización las } \\
\text { posibilidades de formación }\end{array}$ & $\begin{array}{l}\text { Oportunidades de } \\
\text { acceso } \\
\text { Deserción } \\
\text { Apoyo institucional }\end{array}$ \\
\hline Cultural & $\begin{array}{l}\text { Se concentra en el reconocimiento del contexto humano, } \\
\text { costumbres, valoraciones. }\end{array}$ & $\begin{array}{l}\text { Actividades } \\
\text { Grupos a los que } \\
\text { pertenece } \\
\text { Contexto familiar }\end{array}$ \\
\hline Económica & $\begin{array}{l}\text { Se relaciona con las oportunidades de inserción laboral que } \\
\text { genera, la articulación con el sector productivo, el } \\
\text { reconocimiento del entorno económico en que se desarrolla. }\end{array}$ & $\begin{array}{l}\text { Sectores económicos } \\
\text { en desarrollo } \\
\text { Necesidades y } \\
\text { expectativas del sector } \\
\text { productivo }\end{array}$ \\
\hline
\end{tabular}

La revisión de diferentes autores que definen y delimitan el concepto de pertinencia, bajo los enfoques categoriales, relacionados en la Tabla 1, permitió definir el concepto de pertinencia que se abordará para el análisis de los programas del Modelo SENA de Intervención Integral. Por otro lado, en análisis de datos cualitativos y cuantitativos colectados implicó el desarrollo de dos etapas alcanzadas gracias al trabajo de campo realizado.

La primera consistió en la aplicación de instrumentos experimentales, para los cuales fue necesario que los aprendices firmaran un consentimiento informado en el que se les aseguró que sus decisiones fueron completamente anónimas y solo serían de uso investigativo, adicionalmente se les informó que sus decisiones serían absolutamente reales y son incentivadas a través de puntos que podían canjear después por materiales como cuadernos, lapiceros o demás útiles escolares.

Se adoptó el juego de bienes públicos para el análisis de las actitudes cooperativas, ya que este juego permite enfrentar a los individuos a un dilema social en el que deben decidir entre el interés individual o social, además este tipo de juegos abarca bienes que no excluyen a otros de su 
consumo, ni el consumo de una persona reduce el nivel de consumo de la otra. (Cárdenas, 2004; Ostrom 1999). Este tipo de juegos se han implementado en otros países como Burundi, Sierra Leona, Kosovo, Libia e incluso Colombia para estudiar la conducta de los individuos, la discriminación e inclusos sus normas sociales. (Bogliacino et al 2017; Voors et al 2012; Vollhardt, 2012; Mironova and Whitt, 2016; Hartman and Morse, 2016; Craig and Richeson, 2016; Bauer et al 2016; Gilligan et al 2013).

Continuando con los procedimientos que se llevaron a cabo, cabe mencionar que se les pidió contestar un cuestionario inicial de 28 preguntas, por el cuál recibieron 5000 puntos, esto permitía conocer sus características sociodemográficas, actitudes sociales, físicas y emocionales.

Ahora bien, en el juego los participantes recibieron 15000 puntos al inicio de la actividad y se les dijo que iban a ser emparejados al azar con otra persona y la única información disponible que tenían de esa persona es que podía ser una víctima del conflicto, un excombatiente o alguien de población vulnerable, teniendo en cuenta eso debían decidir cuánto enviar a una cuenta grupal en la que por cada 5000 puntos, los investigadores pondrían 2000 puntos adicionales y al final, el total de puntos existentes en la cuenta grupal se dividía entre los dos jugadores. Cabe aclarar que las ganancias de cada uno dependían absolutamente de las decisiones que tomarán y correspondían a la suma de lo que cada individuo dejará en su cuenta individual más lo que ganarán en la cuenta grupal. Por otro lado, se utilizó el método estratégico (Selten, 1967) para capturar las actitudes cooperativas, favoritismos y estereotipos de los participantes respecto a los tipos de población a los que pertenecen los individuos con los que potencialmente son emparejados.

La segunda etapa se llevó a cabo a través de la implementación de entrevistas a los actores que fueron parte del proceso a partir de la aplicación de un muestreo no probabilístico que convino muestreo por conveniencia y el muestreo por avalancha o bola de nieve. El tipo de entrevista aplicada fue semiestructurada, ya que permitió contar con un cuestionario con preguntas principales y con la posibilidad de introducir nuevas preguntas, dependiendo del rumbo que tomaba la entrevista, de la información que aporte el informante o de la necesidad específica del investigador (Kvale, 2011).

De este modo, con base en las categorías definidas en la construcción teórica y estado del arte, se diseñó un instrumento para cada tipo de actor, que abordaba la temática y categoría definida. Como actores clave se definieron aquellos quienes principalmente intervienen en el proceso de formación como instructores, aprendices y funcionarios. Las cantidades de entrevistas se definieron conforme a la cantidad de población inmersa en el proceso de la prueba piloto de acuerdo con la implementación de muestreo por conveniencia y bola de nieve. A continuación, se describen la cantidad de entrevistas realizadas por actor y factor de inclusión: 
Tabla 2. Número de entrevistas aplicadas por actor y según factor de inclusión

\begin{tabular}{lll}
\hline Actor Clave & Factores de inclusión & Cantidad \\
\hline \multirow{2}{*}{ Aprendices } & Pertenecientes a los grupos en formación dentro & 25 (5 por cada grupo) \\
& del proyecto & \\
& Quienes imparten formación a los aprendices de & $12 \quad(4 \quad$ por cada grupo, \\
Instructores & los grupos en formación dentro del proyecto, & incluyendo el líder $)$ \\
& incluyendo los líderes de grupo & \\
& Coordinadores académico y misional & 2 (académica y misional) \\
Funcionarios & Bienestar & 1 trabajadora Social \\
& Equipo de apoyo pedagógico & 3 pedagogos \\
& Contrato de aprendizaje & 1 profesional \\
\multirow{2}{*}{ Total, entrevistas aplicadas } & $\mathbf{4 4}$ \\
\hline
\end{tabular}

\section{RESULTADOS Y DISCUSIÓN}

\subsection{Análisis multidimensional de la pertinencia de programas de formación ofertados bajo} el Modelo SENA de Intervención Integral (MSII)

Teniendo en cuenta las categorías definidas para el análisis documental de información secundaria sobre el concepto de pertinencia, desde diferentes percepciones, el enfoque que se aborda en esta investigación es un enfoque integral capaz de reconocer la relación teoría-practica (PEI - propuesta curricular - practica curricular) y la relación del proceso formativo y la sociedad, pero también, su relación con los aportes e interacciones políticos culturales, económicos, pedagógicos, reconociendo las dinámicas de retroalimentación que deben existir entre las instituciones educativas y el Estado, el sector productivo, los sectores sociales y los sistemas de valores de una sociedad (Malagón, 2006).

En este sentido, el enfoque que se asume en el estudio integra el concepto de pertinencia en relación con ocho dimensiones propuestos en el seminario "Hacia una Agenda de la Educación Superior en Colombia" promovido por la Asociación Colombiana de Universidades (ASCUN) en 1997. Ahora bien, se consolidaron dichas dimensiones en tres componentes, de acuerdo con los enfoques categoriales planteados inicialmente a partir de lo expuesto por Malagón (2006) y Tunnermann (2010). 
Tabla 3. Consolidación de componentes para el análisis de la pertinencia social y educativa.

\begin{tabular}{|c|c|c|c|}
\hline Dimensiones & Concepto & $\begin{array}{l}\text { Enfoques } \\
\text { categoriales }\end{array}$ & $\begin{array}{l}\text { Componente } \\
\text { conformado } \\
\text { para análisis }\end{array}$ \\
\hline $\begin{array}{l}\text { Pertinencia de lo } \\
\text { educativo- } \\
\text { pedagógico }\end{array}$ & $\begin{array}{l}\text { Nuevas pedagogías (innovación en el ámbito educativo } \\
\text { pedagógico) que deriven en pertinencia institucional }\end{array}$ & $\begin{array}{l}\text { Educativo- } \\
\text { Pedagógico }\end{array}$ & $\begin{array}{l}\text { Educativo- } \\
\text { Pedagógica }\end{array}$ \\
\hline $\begin{array}{l}\text { Formación integral } \\
\text { del estudiante }\end{array}$ & $\begin{array}{l}\text { Formación que involucre valores, ética social, sentido de } \\
\text { pertenencia a una comunidad, con lo humano, más allá del } \\
\text { dominio cognoscitivo. }\end{array}$ & Social & $\begin{array}{l}\text { Social, Equidad } \\
\text { Social y Cultural }\end{array}$ \\
\hline $\begin{array}{l}\text { Pertinencia de la } \\
\text { equidad social del } \\
\text { desarrollo. }\end{array}$ & $\begin{array}{l}\text { Ampliación social de cobertura, democratización de las } \\
\text { oportunidades de acceso y logro }\end{array}$ & $\begin{array}{l}\text { Equidad } \\
\text { social }\end{array}$ & $\begin{array}{l}\text { Social, Equidad } \\
\text { Social y Cultural }\end{array}$ \\
\hline $\begin{array}{l}\text { Pertinencia } \\
\text { cultural }\end{array}$ & $\begin{array}{l}\text { "Fortalecimiento de la identidad nacional, rescate y } \\
\text { valoración del patrimonio y creatividad cultural y regional, } \\
\text { promoción de múltiples formas de expresión / creaciones } \\
\text { artísticas; oferta cultural libre de diversos grupos } \\
\text { sociodemográficos, diversos grupos de edad" }\end{array}$ & Cultural & $\begin{array}{l}\text { Social, Equidad } \\
\text { Social y Cultural }\end{array}$ \\
\hline $\begin{array}{l}\text { Pertinencia de } \\
\text { evaluar la } \\
\text { pertinencia }\end{array}$ & $\begin{array}{l}\text { Abordar el análisis de la pertinencia de las instituciones, de } \\
\text { los sistemas, frente a sus proyectos, objetivos, necesidades. }\end{array}$ & Institucional & $\begin{array}{l}\text { Económica, } \\
\text { Institucional y } \\
\text { Política }\end{array}$ \\
\hline $\begin{array}{l}\text { Pertinencia con el } \\
\text { resto del sistema } \\
\quad \text { educativo }\end{array}$ & $\begin{array}{l}\text { Cumplir con las responsabilidades de ser guía en materia } \\
\text { educativa frente a todo el sistema. Asumir el papel } \\
\text { conductor en la renovación de todo el sistema educativo. } \\
\text { Formación de personal, investigación socio educativa, } \\
\text { nuevas tecnologías. }\end{array}$ & & $\begin{array}{l}\text { Económica, } \\
\text { Institucional y } \\
\text { Política }\end{array}$ \\
\hline $\begin{array}{l}\text { Pertinencia } \\
\text { política }\end{array}$ & $\begin{array}{l}\text { Capacidad de presentar alternativas, de construir } \\
\text { soluciones, de generar pertinencia social. }\end{array}$ & Político & $\begin{array}{l}\text { Económica, } \\
\text { Institucional y } \\
\text { Política }\end{array}$ \\
\hline $\begin{array}{l}\text { Pertinencia con el } \\
\text { sector productivo }\end{array}$ & $\begin{array}{l}\text { Suplir las demandas de la economía y en la estrecha } \\
\text { relación con el desarrollo científico-tecnológico. }\end{array}$ & Económica & $\begin{array}{l}\text { Económica, } \\
\text { Institucional y } \\
\text { Política }\end{array}$ \\
\hline
\end{tabular}

Fuente: Malagón (2006) y Tunnermann (2010).

\subsubsection{Análisis cuantitativo}

\section{Datos descriptivos de las características sociodemográficas}

En primera instancia, se debe mencionar que las sesiones experimentales se realizaron con 258 aprendices, los cuales conformaban los programas de formación evidenciados en la Tabla No. 2. Dentro de la muestra, la población se clasificó en tres tipos: víctimas del conflicto armado (77), personas en proceso de reincorporación (7) y población vulnerable (174), esta última abarcó a aquellas personas pertenecientes a una minoría étnica, madres cabeza de hogar y discapacitados. En este sentido, tomando el concepto ya definido, Labrunée \& Gallo (2005) consideran que la vulnerabilidad social se manifiesta en aquella población que se ve afectada por cambios económicos, políticos y sociales. Además, Estivill (2003) afirma que dicha vulnerabilidad está 
compuesta por problemáticas como el desempleo, la deserción escolar, escasez de recursos económicos, etc.

Tabla 4. Número de aprendices encuestados

\begin{tabular}{ccc} 
Nivel & $\begin{array}{c}\text { Programa } \\
\text { académico }\end{array}$ & $\begin{array}{c}\text { No. De } \\
\text { aprendices }\end{array}$ \\
\hline \multirow{3}{*}{ Técnico } & Alojamiento Rural & 21 \\
& Manejo Ambiental & 23 \\
& Ventas y Servicios & 27 \\
\hline \multirow{2}{*}{ Cursos } & Cocina & 92 \\
complementarios & Turismo & 29 \\
& Ventas & 66 \\
\hline & Total & 258 \\
\hline
\end{tabular}

Si bien es cierto, el SENA apertura sus programas con el objetivo de generar inclusión social y aportar al bienestar de la comunidad, de acuerdo a los datos obtenidos es posible observar que el $41 \%$ de la población se identifica como mestizos, $29 \%$ como afrocolombianos, el 16\% como blancos, el $8 \%$ como indígenas, el $2 \%$ como mulato y el $4 \%$ restantes no se auto-reconoce en ninguno de los grupos mencionados. En ese mismo sentido el gráfico No. 1 muestra dicha clasificación diferenciando por tipo de población.

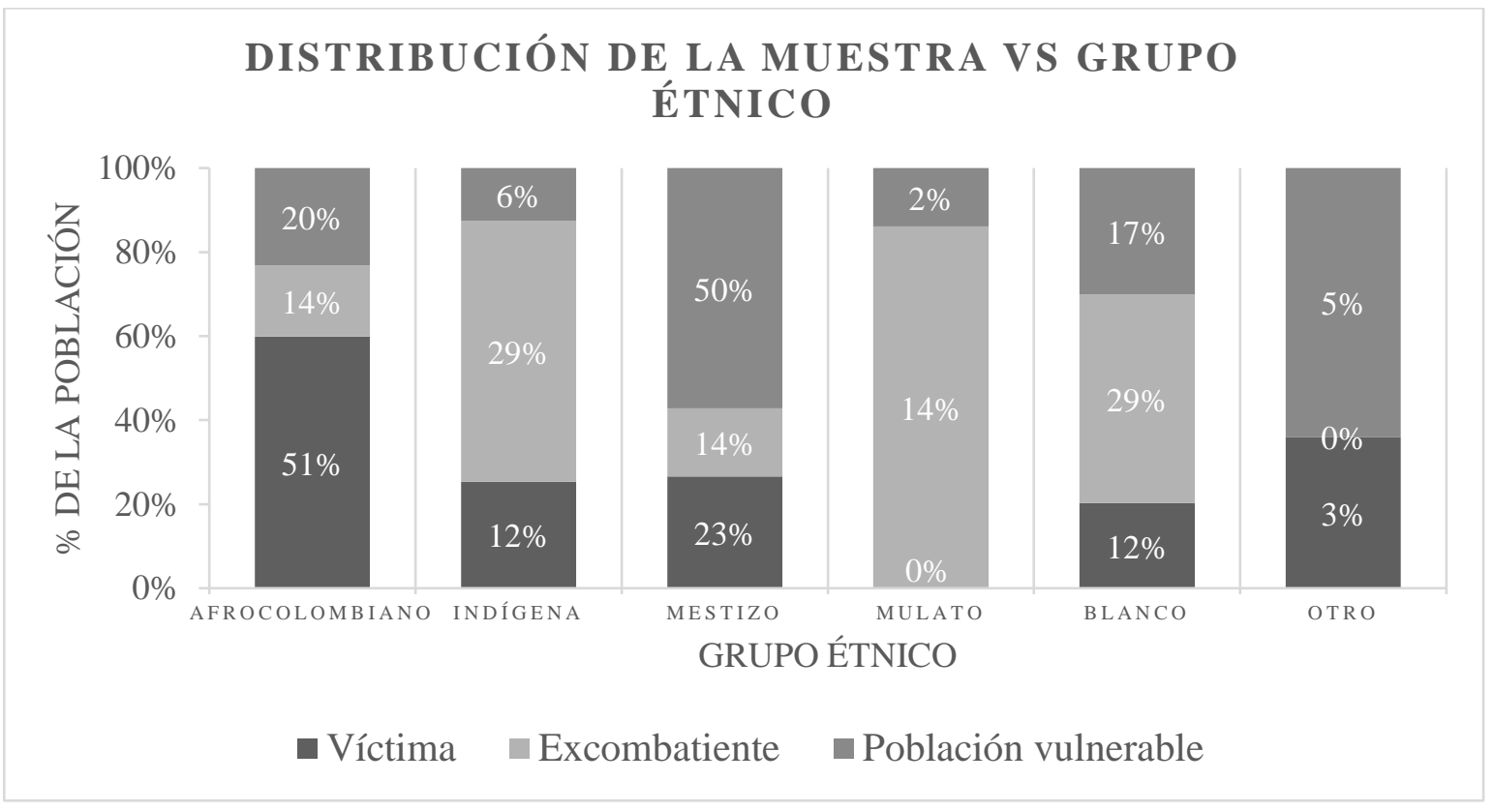

Figura 1 Distribución de la población vs Grupo étnico 
Ahora bien, respecto a diversas características de la muestra se encuentra que la mayoría de los beneficiarios fueron mujeres con un $62,02 \%$ y el 37,98\% fueron hombres. Así mismo, se obtuvo que la edad promedio fue de 33 años, la edad mínima fue de 15 y la máxima de 75 años. Cabe mencionar que en relación al estrato socioeconómico ${ }^{6}$ el $82,75 \%$ de las personas pertenecen al estrato 1 y 2.

Por otro lado, el análisis indica que la población beneficiaria ha estado expuesta a distintos tipos de violencia tanto a nivel individual $(66,15 \%)$ como a nivel familiar $(71,6 \%)$. Sin embargo, aquí es clara la relevancia de involucrar a la población que no ha sido afectada directamente por el conflicto, ya que esto permite cerrar brechas sociales entre la comunidad.

\section{Análisis descriptivo de las actitudes cooperativas y sesgos intergrupales}

De acuerdo con la muestra se encontró que la contribución en promedio es más alta hacia las personas en condiciones de vulnerabilidad $(11219,1)$, seguida por aquellas que son víctimas del conflicto $(10059,8)$ y por último se sitúa la contribución media hacia los excombatientes $(7629,48)$. El gráfico No. 2 muestra los resultados de las contribuciones promedio, en los que se exhibe un sesgo hacia los excombatientes y un favoritismo de la muestra en general por cooperar más con aquellas personas que pertenecen a la población vulnerable.

\footnotetext{
${ }^{6}$ De acuerdo con el Departamento Administrativo Nacional de estadística (DANE) el Estrato socioeconómico hace referencia a la clasificación de los hogares en Colombia, con base en las características de las viviendas y su entorno urbano o rural, en el que se relaciona la vivienda con el entorno como una representación de un modo socioeconómico. Esta clasificación consta de seis estratos que pueden ser vistos como una aproximación a la diferencia socioeconómica jerarquizada (de pobreza a riqueza o viceversa). Así, los estratos 1, 2 y 3 corresponden a estratos bajos que albergan a los usuarios con menores recursos y los estratos 4,5 y 6 corresponden a estratos altos que albergan a los usuarios con mayores recursos económicos.
} 


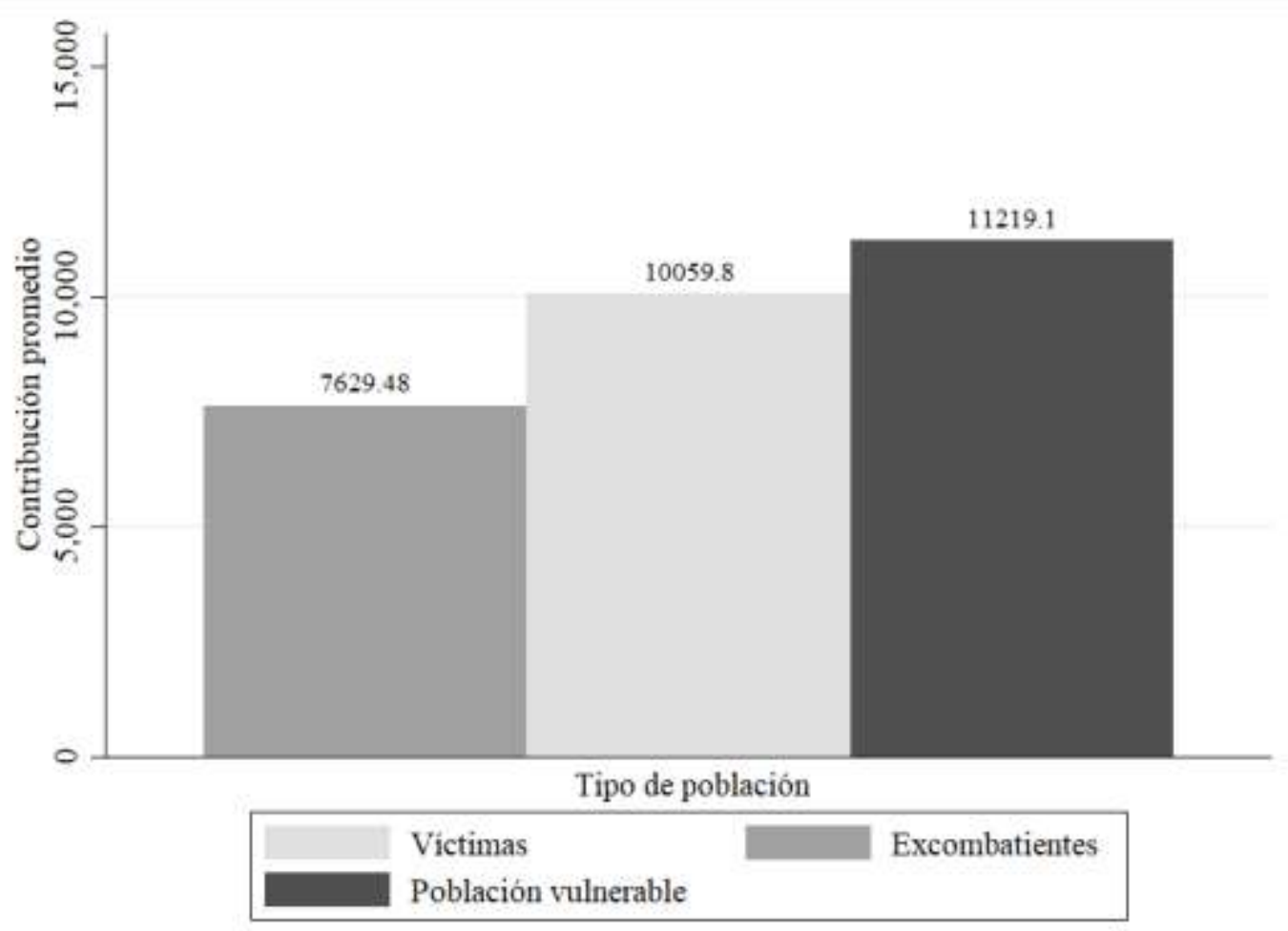

Figura 2 Distribución de la población vs Grupo étnico

Para finalizar, los participantes afirman en una escala de 1 a $5^{7}$ estar dispuestos a emprender nuevos proyectos en promedio en 4,43, mientras que cuando se les preguntó por sus capacidades para liderar, el valor promedio señalado por los participantes es tan solo de 2,67 en la escala de 1 a 5 . Lo cual, indica que es probable que sea necesario potencializar o encaminar la enseña en el desarrollo de habilidades entorno al liderazgo.

\subsubsection{Análisis cualitativo}

En este apartado se relacionarán las percepciones y conceptos derivados de las entrevistas aplicadas a aprendices, instructores, funcionarios y directivos, alrededor de los componentes definidos para el análisis de la pertinencia social y educativa de los programas impartidos a través del Modelo SENA de Intervención Integral.

\section{Pertinencia Educativo-pedagógico}

Enfocando el análisis en el componente educativo-pedagógico es posible pensar que en cuestiones de metodología los instructores se rediseñaron, de tal forma que sus estudiantes adoptaran los conocimientos de una forma eficaz, teniendo en cuenta el tipo de población se debió buscar la

\footnotetext{
${ }^{7}$ La escala señalada de 1 a 5 en estas preguntas indicaba el nivel de frecuencia con la que el participante realizaría una acción, siendo 1 nunca y 5 muy frecuentemente.
} 
manera más adecuada logrando que la pedagogía implementada rindiera los frutos esperados al final de la formación, dado que se presentaron deficiencias a nivel académico.

Respecto al tema, el pronunciamiento de parte de los instructores fue:

“(...) se utilizan estrategias más didácticas, que sólo teoría, libros, no, entonces videos, hacíamos una lectura, pero entonces no saben leer muy bien, entonces ya tocaba hacerles más bien la comprensión de lectura, o sea, ya el aprendizaje tiene que ser más guiado, no es tan autónomo como uno lo puede hacer con un muchacho que ya tiene su bachiller, sabe leer, sabe escribir, ellos no sabían escribir bien, algunos no sabian leer, y que no sabían expresarse en público” (Entrevista a instructor 1).

Por consiguiente, enseñar acarrea una responsabilidad no solo en el amplio conocimiento que debe poseer un instructor sino también en la estrategia que utilice para extenderlo a sus aprendices, puesto que la teoría es un punto relevante pero la metodología con la que se desarrolle es clave. Los instructores comparten este pensar, un ejemplo claro se obtuvo de una de las entrevistas en las que se afirma que: “(...) compartir con ellos en primer lugar en aspectos muy de la realidad, no tanto el contexto teórico de los programas, sino aterrizarlos a ellos en situaciones reales, acercándolos a la realidad, porque además de otra forma es difícil enseñar, o sea, con ellos se debe ponerse en los zapatos y aterrizar en el plano de ellos” (Entrevista a instructor 2).

De acuerdo con lo anterior, el SENA ha desarrollado una estrategia desde la parte administrativa de cuál es la mejor manera de atender a este tipo de población. La respuesta fue la siguiente por parte de un funcionario:

“(...) es un modelo integral que busca que todos los servicios que el Sena ofrece, se den en diferentes momentos, pero articulados, ejemplo, damos formación, pero también tenemos el desarrollo de las habilidades blandas por medio de deporpaz, pero también a su vez se está trabajando el componente de emprendimiento, pero también el componente de empleabilidad y ya los componentes que tienen que ver con investigación, para resumir, es un modelo que articula los diferentes servicios que el Sena brinda, dirigido especialmente a la población, no de manera independiente sino articulada" (Entrevista a funcionario 1)

A saber, la opinión de los aprendices es un atributo importante para definir la pertinencia alrededor de lo educativo-pedagógico, ya que la demanda y la oferta académica es de gran relevancia para el análisis, dada la relación entre estas con el interés y éxito dentro de la formación. Respecto a 
esto, la evidencia permite afirmar que para los aprendices ha sido gratificante no solo el contenido técnico del programa sino también sus componentes adicionales. Un ejemplo de esto es:

“(...) todas las competencias me han parecido buenísimas, porque todas tienen puntos de vista diferentes y los profesores son buenos. Todas me parecen interesantes. Si me ponen a escoger una sería como la más importante sería ética, me parece buenísimo porque ahí las personas a pesar de ser inteligentes deben tener valores definidos para brindar un buen servicio al cliente. Y de menor importancia, no. Todas son buenas. Hasta educación física nos están haciendo batalla de fuerza para ver que el trabajo en equipo es importante y la unión es importante. Ayuda a la cooperación, trabajo en equipo" (Entrevista de aprendiz 1)

Finalmente, cabe recalcar que existen una serie de necesidades claves en el proceso, tales como: un mejoramiento en la orientación vocacional que permita una adecuada selección del programa de formación, un diagnóstico inicial de las condiciones de la población a nivel educativo y social, seguida de una nivelación de los conocimientos preliminares necesarios para cursar un curso técnico y por último, quizás una estrategia estandarizada de la metodología de enseñanza que combine la teoría con la práctica.

\section{Pertinencia Social, Equidad Social y Cultural}

De acuerdo con diversas entrevistas a instructores y funcionarios, es posible considerar que la población objetivo se encuentra en una situación de alejamiento frente a los procesos formativos, la interacción con la institucionalidad y la sociedad misma, de este modo los aprendices presentan problemas para adaptarse a las dinámicas institucionales, a cumplir tiempos y plazos, a comprender los procedimientos establecidos para los diferentes trámites al interior del SENA e incluso la forma en la que se relacionan, se dirigen a otros o reconocen la autoridad. Lo cual requiere un apoyo adicional de parte del área de Bienestar, en el que se trabajen actitudes de servicio al cliente y demás, junto con el desarrollo del ser. En relación con esto, se evidenciaron ciertos comportamientos que los funcionarios e instructores manifestaron, como los siguientes:

“(...) a veces es difícil que ellos como que reciban como las órdenes, tuvimos algunos que les costaba, les daba dificultad adaptarse a unos horarios, adaptarse a de pronto al ámbito laboral, les daba dificultad, entonces apenas llevaban un mes y no es que no me gusta cómo me tratan, no me gusta cómo me miran, no me gusta como esto" (Entrevista a funcionario 2).

“(...) la forma de expresarse es muy brusco, es muy tosco, tiende a ser grosero, no con palabras grosera, pero sí la forma en el tono de voz y esas cosas, esa fue la primera 
dificultad que yo tuve, pero ya como a las 2 semanas que uno ya empieza a observarlos, uno se da cuenta que es que ellos no son groseros, siempre se criaron asi'" (Entrevista a instructor 2).

A su vez, se reconoce que el SENA dentro del proceso generó un impacto a nivel personal en los aprendices, los instructores por su parte percibieron que para ellos el SENA es más que una institución: "dentro de las cosas que ellos decían era que en la casa no encontraban una familia, y esa familia la podían encontrar ahí" (Entrevista a instructor 3).

De manera similar, otro instructor considera frente al tema que:

“(...) el SENA era una oportunidad como de salir de ese mundo, de esa esfera donde ellos estaban, y además no sólo la oportunidad de estudiar, sino también de que ellos con emprender pueden montar su empresa, por ejemplo, hay una aprendiz que ella quiere montar su hostal, entonces con el proyecto se le ayudó a realizar el proyecto, y ahora está haciendo el proceso para hacerlo pasar al Sena emprender, o de igual forma buscar otras formas de financiación para ella superarse, entonces no sólo se les enseñó para que fueran trabajadores, sino que también para que pudieran emprender, entonces es una oportunidad, y una forma de ver diferente las cosas, como salirse de ese mundo, ese contexto donde ellos normalmente están..." (Entrevista a instructor 1).

Así mismo, la trabajadora social manifiesta que percibió en los aprendices que:

“(...) se les abre una puerta de esperanza para el mundo laboral y con ello va la calidad de vida de sus familias, son personas de las que yo hablé, con muchos de los que yo hablé, me contaban su historia de vida, identifiqué que es una oportunidad de vida, ellos sienten que es un momento para que ellos empiecen de nuevo, oportunidades sociales, porque ya se sienten personas que le pueden ayudar a un vecino, a un compañero, y crear una estrategia positiva” (Entrevista a funcionario 2).

Así pues, se presentaron resultados relevantes en el proceso de formación, reconocidos por los instructores, uno de ellos fue expresado así:

“(...) pero una mujer muy guerrera, se superó, ella al principio no podía hablar en público, le daba mucha pena, no sabía expresarse, me decía que no era capaz de hacerlo, pero muy inteligente, ella sabía inclusive más que los compañeros, pero cuando pasaba adelante se bloqueaba totalmente, tenía una autoestima muy baja, y cuando salimos, cuando fue la presentación del proyecto de ellos, yo me quedé aterrada de ver cómo se expresó, pero eso no se logra sin la ayuda de bienestar, sin que los instructores hubieran estado alli" (Entrevista a instructor 3) 
Desde la parte social, se observa que la población intervenida ha vivido y vive situaciones afectivas y familiares de gran dificultad. Esto influye en la capacidad de confianza, interacción y cohesión con el grupo. Las personas que participaron en el proyecto viven aún en ambientes de conflicto social, permanentemente se escucha que sus amigos o familiares fueron asesinados, que ellos mismos fueron atacados en su integridad física, que sus familias los rechazan o no les prestan apoyo, entre otros.

En relación con lo anterior, los datos obtenidos de la encuesta permiten observar que el $81,69 \%$ de la muestra ha sido testigo o alguno de sus familiares han sido víctimas de la violencia, mientras que, el 78,87\% ha estado expuesto a la violencia a nivel individual. En este sentido, uno de los instructores expone una historia que remarcó en su memoria: “(...) a ella le mataron al esposo delante de ellos y la sacaron, que también la violaron, esa historia a mí me pareció durísima” (Entrevista a instructor 3).

En definitiva, resulta necesario generar un fortalecimiento de "competencias transversales o blandas" que coadyuven a la readaptación de la población a las dinámicas sociales formales o institucionales, esto de la mano del acompañamiento emocional individual y grupal, así como, el seguimiento sistemático de los procesos de cada aprendiz. Así pues, se precisa que el proceso institucional reconozca y articule como parte del proceso formativo y administrativo, las condiciones y necesidades especiales de esta población.

\section{Pertinencia Económica, Institucional y Política}

En términos económicos, la población atendida en su mayoría no presenta una actividad económica definida ni una vocación identificada, de este modo la elección del programa a estudiar se ha hecho más desde el gusto por el título del programa, lo que se imaginan que podría ser o por la disponibilidad de cupo. Igualmente, no poseen un empleo o labor, ni una experiencia considerable en esta actividad. En otras palabras, esta población no trae experiencia laboral, razón por la cual, se le dificulta hacer un análisis integral para la elección del programa de estudio.

Teniendo en cuenta que se trata de población especial, la búsqueda de empleadores o patrocinadores se dificulta en mayor grado, puesto que, aunque socialmente se reconoce la necesidad de ofrecer oportunidades a las personas en esta situación como mecanismo para mejorar la calidad de vida y superar problemas de violencia y conflicto, en la práctica en general, el resto de la población, en especial los empresarios, no se comprometen en la labor de abrir esas oportunidades en sus empresas. 
Respecto al tema, uno de los funcionarios del SENA dice:

“(...) en este momento estamos buscando el patrocinio, porque ha sido de las cosas más difíciles, si ellos hubieran tenido el patrocinio desde el inicio, no hubieran existido tantas decepciones, porque ellos hubieran tenido un ingreso para que ellos se hubieran podido sustentar, pero pues esta es la hora y no hemos podido conseguir patrocinio, se ha hecho la gestión, pero como el programa es tan nuevo, entonces sí es un poco más complejo, pero yo digo que si seguimos trabajando sobre esos hoteles rurales que hay acá en Cali y sobre que se den a conocer, todo el contexto de lo que el Sena está haciendo con ellos, que es una oportunidad de vida que estamos dando, no es sólo un aprendiz más, es una persona que se está ayudando a salir de un entorno de guerra, de dolor, entonces eso sería muy bueno" (Entrevista a funcionario 3).

De igual modo, se observa que la falta de experiencia laboral, sumado a un cierto desconocimiento de algunas habilidades o herramientas básicas para el relacionamiento social, tales como el vocabulario, la presentación personal, los modales, la disposición del cuerpo, las formas de responder, entre otras, hacen que los aprendices no logren sortear con éxito las entrevistas para ingreso.

Desde la visión económica, los recursos de este tipo de población son escasos, lo que afecta sus posibilidades materiales para el desarrollo de alguna actividad que rompa la cadena de exclusión social. No cuentan con un empleo ni con un ingreso suficiente para cubrir sus necesidades básicas, de manera que no alcanza a cubrir la alimentación y el transporte para asistir a formación, menos solventar otros gastos como uniformes, fotocopias, etc.

A propósito de esto, los instructores declaran:

“(...) vienen y viven en situaciones muy duras, qué hacían ellos para poder ir a estudiar, como el horario muchas veces era jornada completa, trabajaban por las noches muchas veces, había una señora que vendía café y jugos, desde las 3 de la mañana hasta las 5 o 6, se cambiaba e iba a estudiar, los fines de semana trabajaba como empleada doméstica, cualquier cosa, lo que les salga, ellos trabajan en eso, porque tienen sus hijos y a veces no tienen cómo mantenerlos, y los muchachos jóvenes que tenían sus papás, encontraban que no los ayudaban, ni siquiera saben qué es lo que hacen, entonces son situaciones complejas." (Entrevista a instructor 1)

En pocas palabras, se precisa del reconocimiento, compromiso y aporte de todos los actores involucrados en la finalidad de obtener procesos integrales; con lo cual se garantizaría la sustentabilidad económica de los aprendices y el acceso a la empleabilidad, conforme lo descrito en líneas anteriores. 


\section{CONCLUSIONES}

A partir de los resultados y discusión generada, en la Tabla 5 se describen los aspectos que evidencian la pertinencia de los programas en cada uno de los componentes propuestos para el análisis y según los conceptos emitidos por los actores involucrados en el proceso de formación.

Tabla 5 Síntesis de análisis de la pertinencia según componentes establecidos

\begin{tabular}{|c|c|c|c|}
\hline & Instructores & Funcionarios & Aprendices \\
\hline $\begin{array}{l}\text { Pertinencia Educativo- } \\
\text { pedagógico }\end{array}$ & $\begin{array}{l}\text { La pertinencia se } \\
\text { evidencia en el } \\
\text { rediseño de estrategias } \\
\text { metodológicas y } \\
\text { pedagógicas por parte } \\
\text { de los instructores para } \\
\text { que los contenidos } \\
\text { sean relevantes para } \\
\text { los aprendices. }\end{array}$ & $\begin{array}{l}\text { El modelo de formación es } \\
\text { pertinente toda vez que logra } \\
\text { abordar el ser, aprender y hacer, } \\
\text { integrando en su implementación } \\
\text { los diferentes servicios que ofrece } \\
\text { el SENA teniendo en cuenta la } \\
\text { particularidad de la población } \\
\text { objetivo con el componente } \\
\text { DEPORPAZ. }\end{array}$ & $\begin{array}{l}\text { Gran aceptación tanto } \\
\text { de los contenidos } \\
\text { técnicos, transversales } \\
\text { y de DEPORPAZ } \\
\text { como contribución a } \\
\text { su formación integral. }\end{array}$ \\
\hline $\begin{array}{l}\text { Pertinencia Social, } \\
\text { Equidad Social y } \\
\text { Cultural }\end{array}$ & $\begin{array}{l}\text { Formación como } \\
\text { medio para superar } \\
\text { temores que impiden } \\
\text { el desarrollo técnico a } \\
\text { raíz de historias de } \\
\text { vida complejas, } \\
\text { gracias a un adecuado } \\
\text { acompañamiento. }\end{array}$ & $\begin{array}{l}\text { Aporte a la creación de } \\
\text { oportunidades para ingresar al } \\
\text { mundo laboral. }\end{array}$ & $\begin{array}{l}\text { Reconocimiento del } \\
\text { SENA como } \\
\text { oportunidad de } \\
\text { crecimiento y } \\
\text { desarrollo. }\end{array}$ \\
\hline $\begin{array}{l}\text { Pertinencia Económica, } \\
\text { Institucional y Política }\end{array}$ & $\begin{array}{l}\text { Dificultad por jornadas } \\
\text { de formación } \\
\text { extendidas (mañana y } \\
\text { tarde) debido a la } \\
\text { situación económica, } \\
\text { pues muchos } \\
\text { necesitaban trabajar } \\
\text { para su sustento. }\end{array}$ & $\begin{array}{l}\text { Dificultad para encontrar contrato } \\
\text { de aprendizaje, como forma de } \\
\text { apoyo para que los aprendices } \\
\text { permanezcan en la formación. }\end{array}$ & $\begin{array}{l}\text { Pese a las facilidades } \\
\text { en términos de acceso, } \\
\text { permanecer en } \\
\text { formación es difícil } \\
\text { por la situación } \\
\text { económica de la } \\
\text { mayoría de los } \\
\text { aprendices }\end{array}$ \\
\hline
\end{tabular}

De acuerdo con el análisis, se establece que los programas de formación son pertinentes respecto a los componentes: educativo-pedagógico y la pertinencia social, equidad social y cultural. Así pues, para generar un impacto en el componente de la pertinencia económica, institucional y política, es vital establecer estrategias de articulación con los empresarios, como posibles patrocinadores o contratantes de estos aprendices en momentos previos al desarrollo del programa. 
Estas estrategias deben de ir acompañadas de jornadas de sensibilización en las que se genere mayor conciencia y compromiso por parte del sector productivo frente a la lucha contra la pobreza y la construcción de paz. De este modo, se garantiza mínimamente, que los aprendices tengan la oportunidad, al menos, de hacer su etapa práctica. Cabe resaltar, que este punto hace parte de una propuesta presentada a la institución como la segunda fase de la consolidación de Modelo SENA de Intervención Integral.

Ahora bien, dado que el área de Bienestar fue indispensable para que el proceso de formación de los aprendices fuese exitoso, se considera necesario que con la experiencia previa obtenida en esta intervención se diseñe un modelo de atención de este tipo de población, en el cual, se reconozcan previamente las necesidades específicas de esta población y se atiendan de manera planificada y articulada al proceso formativo.

Finalmente, un punto clave es el diseño de actividades extracurriculares y didácticas que les permitan a los aprendices tener espacios de liberación de tensiones, pero a su vez tener elementos prácticos de motivación y estímulo para continuar con su proceso. En este tipo de población se observa que el resultado fundamental está encaminado en suscitar y fortalecer su liderazgo y un sentido de pertenencia a la institución, al grupo y a su proceso, como mecanismo para lograr generar un compromiso con la obtención de resultados satisfactorios tanto a nivel académico como a nivel laboral.

En términos de la caracterización y análisis de comportamiento debemos esclarecer que los excombatientes no son parte de las pruebas estadísticas, dado que las observaciones carecen de poder estadístico, adicionalmente, los resultados aquí presentados son de carácter descriptivo y no evalúan causalidad ni inferencia. Se encontró que los individuos reconocen sus habilidades para emprender, pero no sus capacidades de liderar, lo cual no difiere significativamente entre víctimas y población vulnerable.

Respecto a sus actitudes cooperativas se hace visible cuantitativamente un sesgo hacia los excombatientes, tomando como referencia las medias de las contribuciones a la cuenta grupal dependiendo de cada tipo de población, la muestra afirma querer cooperar, en promedio, con alguien de población vulnerable un 75\% de su dotación inicial (15.000), con una víctima este porcentaje es del $67 \%$ y con un excombatiente es del 51\%. Sin embargo, la presente investigación no puede dar cuenta del posible efecto sobre los individuos la transición por el modelo de intervención MSII.

De acuerdo con diversas entrevistas a instructores y funcionarios, es posible considerar que la población objetivo se encuentra en una situación de alejamiento frente a los procesos formativos, la interacción con la institucionalidad y la sociedad misma, razón por la cual los aprendices 
presentan problemas para adaptarse a las dinámicas institucionales, cumplir tiempos y plazos, comprender los procedimientos establecidos para los diferentes trámites al interior del SENA e incluso la forma en la que se relacionan, se dirigen a otros o reconocen la autoridad (Estivill, 2003; Labrunée \& Gallo, 2005).

También es importante señalar que, gracias al análisis documental de información secundaria sobre el concepto de pertinencia desde diferentes dimensiones, se lograron consolidar factores y características en tres componentes para el análisis de la pertinencia:

Educativo-Pedagógica,

Social, Equidad Social y Cultural

Económica, Institucional y Política

Estos componentes, en su conjunto permitieron reconocer las dinámicas de retroalimentación que deben existir entre las instituciones educativas y el Estado, el sector productivo, los sectores sociales y los sistemas de valores de una sociedad (Malagón, 2006; 2009).

\section{REFERENCIAS BIBLIOGRÁFICAS}

Bauer, M., Blattman, C., Chytilová, J., Henrich, J., Miguel, E., \& Mitts, T. (2016). Can war foster cooperation? The Journal of Economic Perspectives, 30(3), 249-274.

Bogliacino, F., Grimalda, G., Ortoleva, P., \& Ring, P. (2017). Exposure to and recall of violence reduce short-term memory and cognitive control. Proceedings of the National Academy of Sciences, 114(32), 8505-8510.

Cárdenas, J. C. (2004). Regulaciones y normas en lo público y lo colectivo: exploraciones desde el laboratorio económico. Documentos CEDE.

Craig, M. A., \& Richeson, J. A. (2016). Stigma-based solidarity: Understanding the psychological foundations of conflict and coalition among members of different stigmatized groups. Current Directions in Psychological Science, 25(1), 21-27.

Dias, J. (2008). Calidad, Pertinencia y Responsabilidad Social de la Universidad Latinoamericana y Caribeña. En Tendencias de la Educación Superior en América Latina y el Caribe. doi: https://doi.org/10.1016/j.jpowsour.2007.06.235

Díaz, J. R., \& González, R. R. (2001). Influencia de la práctica de deporte para la adquisición de habilidades sociales en los adolescentes. Aula abierta, (78), 29-46. Recuperado de https://dialnet.unirioja.es/servlet/articulo?codigo=209146

Estivill, J. (2003). Concepts and strategies for combating social exclusion: An overview. Strategies and Tools against Social Exclusion and Poverty Programme.

García, F. (2002). Currículum y pertinencia. Docencia Universitaria, 3(2), 107-123. 
García, L. M., Gutiérrez, D., González, S. y Valero, A. (2012). Cambios en la empatía, la asertividad y las relaciones sociales por la aplicación del modelo de instrucción educación deportiva. Revista de Psicología del Deporte, 21(2),321-330. Recuperado de: https://www.redalyc.org/articulo.oa?id=2351/235126897012

Gibbons, M. (1998). Pertinencia de la educación superior en el siglo XXI. Education, Human Development Network, World Bank.

Gilligan, M. J., Mvukiyehe, E. N., \& Samii, C. (2013). Reintegrating rebels into civilian life: Quasi-experimental evidence from Burundi. Journal of Conflict Resolution, 57(4), 598626.

Harris, I., \& Mische, P. M. (2004). Environmental Peacemaking, Peacekeeping, and Peacebuilding: Integrating Education for Ecological Balance and a Sustainable Peace. Educating for a culture of social and ecological peace, 169-182.

Hartman, A., \& Morse, B. (2016). Violence, Empathy, and Altruism: Evidence from the Ivoiran Refugee Crisis in Liberia. Browser Download This Paper.

Higuera, L. (2018). Pertinencia de la cátedra para la paz en instituciones de educación superior. (Tesis de Especialización). Universidad Militar Nueva Granada. Bogotá.

Kvale, S. (2011). Las entrevistas en investigación cualitativa. Ediciones Morata.

Labrunée, M. E., \& Gallo, M. E. (2005). Vulnerabilidad social: el camino hacia la exclusión.

Ley 1064 de 2006: Por la cual se dictan normas para el apoyo y fortalecimiento de la educación para el trabajo y el desarrollo humano establecida como educación no formal en la Ley General de Educación. Cong. (26 de julio de 2006)

Malagón Plata, L. A. (2006). La pertinencia en la educación superior: elementos para su comprensión Consideraciones generales. Revista de la Educación Superior.

Malagón Plata, L. A. (2007). Currículo y pertinencia en la Educación Superior. Bogotá, Colombia: Cooperativa Editorial Magisterio.

Malagón Plata, L. (2009). La vinculación Universidad-Sociedad desde una perspectiva social. Educación y Educadores, 9(2), 79-93. Recuperado de: https://educacionyeducadores.unisabana.edu.co/index.php/eye/article/view/664

Martuscelli, J. \& Martínez, C. (2002). Problemas de la Pertinencia de la Educación Superior en el Mercado Laboral. Memoria del Tercer Congreso Nacional y Segundo Internacional Retos y Experiencias de la Universidad, 6-9 de noviembre, Toluca, México: Universidad Autónoma del Estado de México.

Mendoza Guzmán, Y. M. (2018). Evaluación de la pertinencia social del currículo del programa de inclusión educativa para adultos de la Institución Educativa Integrada La Candelaria (tesis de maestría). Universidad Externado, Bogotá.

Mironova, V., \& Whitt, S. (2016). Social Norms after Conflict Exposure and Victimization by Violence: Experimental Evidence from Kosovo. British Journal of Political Science, 1-17. 
Moscoso, D., Pérez, A., Muñoz, V., González, M., y Rodríguez, L. (2013). El deporte de la libertad. ANDULI, Revista Andaluza de Ciencias Sociales, (11), 55-69.

Observatorio de Procesos de Desarme,Desmovilización y Reintegración. (2009). Las Universidades y la reintegración a la vida civil en Colombia. Universidad Nacional, Facultad de Ciencias Económicas. Recuperado de: https://repositorio.unal.edu.co/bitstream/handle/unal

Ostrom, E. (1998). A Behavioral Approach to the Rational Choice Theory of Collective Action: Presidential Address, American Political Science Association, 1997. American Political Science Review. DOI: https://doi.org/10.2307/2585925

Peña, A. Q. (2006). Metodología de investigación científica cualitativa. Psicología: Tópicos de actualidad, 47-84.

Ramírez, A. A. (2010). La evaluación como herramienta para mejorar los procesos educativos de poblaciones socialmente vulnerables. Revista Iberoamericana de Educación, 53(5). Recuperado de: https://rieoei.org/historico/deloslectores/3778Ramirez.pdf

Tajfel, H., y Turner, J. (1979). An Integrative Theory of Intergroup Conflict. The Social Psychology of Intergroup Relations. https://doi.org/10.1016/S0065-2601(05)37005-5

Tajfel, H., y Turner, J. C. (1986). The Social Identity Theory of Intergroup Behavior. En Psychology of Intergroup Relations. doi: https://doi.org/10.1111/j.17519004.2007.00066.x

Terigi, F. (2009). "El fracaso escolar desde la perspectiva psicoeducativa" en Revista Iberoamericana de Educación, no. 50, Madrid, OEI. doi: https://doi.org/10.35362/rie500659

Tunnermann, C. (2000). Pertinencia social y principios básicos para orientar el diseño de políticas de educación superior. Educación Superior y Sociedad.

UNESCO. (1998). La Educación superior en el siglo XXI: Visión y acción. Conferencia Mundial sobre la Educación Superior: la educación superior en el siglo XXI - visión y acción.

UNESCO, L. (2008). Reflexiones en torno a la evaluación de la calidad educativa en América Latina y el Caribe.

Santos, M. (1998). Evaluar es comprender. Buenos Aires: Editorial Magisterio.

SENA, (s.f.). Misión y Visión SENA. Recuperado de http://www.sena.edu.co/esco/sena/Paginas/misionVision.aspx

Selten, R. (1965). Die Strategiemethode zur Erforschung des eingeschränkt rationalen Verhaltens im Rahmen eines Oligopolexperimentes. Seminar für Mathemat. Wirtschaftsforschung u. Ökonometrie.

Sutz, J. (1997). La universidad latinoamericana y su pertinencia: elementos para repensar el problema, en Revista Quantum/CCEEA, 9. 
Vollhardt, J. R. (2012). Collective victimization. Oxford handbook of intergroup conflict, 136157.

Voors, M., Nillesen, E. E., Verwimp, P., Bulte, E. H., Lensink, R., \& Van Soest, D. (2010). Does conflict affect preferences? Results from field experiments in Burundi.

\section{Conflicto de intereses / Competing interests:}

Los autores declaran que no incurre en conflictos de intereses.

\section{Rol de los autores / Authors Roles:}

Gustavo Rincón. conceptualización, curación de datos, análisis formal, adquisición de fondos, investigación, metodología, administración del proyecto, recursos, software, supervisión, validación, visualización, escritura preparación del borrador original, escritura - revisar \& amp; edición.

Nathaly Jiménez: conceptualización, investigación, metodología, administración del proyecto, recursos, software, supervisión, validación, visualización, escritura - preparación del borrador original, escritura - revisar \& amp; edición.

Michelle Castrillón: conceptualización, curación de datos, supervisión, validación, visualización, escritura preparación del borrador original, escritura - revisar \& amp; edición.

\section{Fuentes de financiamiento / Funding:}

Los autores declaran que no recibió un fondo específico para esta investigación.

\section{Aspectos éticos / legales; Ethics / legals:}

Los autores declaran no haber incurrido en aspectos antiéticos, ni haber omitido aspectos legales en la realización de la investigación. 